Physics in Collision (PIC 2013)

International Journal of Modern Physics: Conference Series

Vol. 31 (2014) 1460285 (13 pages)

(C) The Authors

DOI: $10.1142 / \mathrm{S} 2010194514602853$

\title{
Low energy neutrinos
}

\author{
G. Ranucci \\ Istituto Nazionale di Fisica Nucleare \\ Via Celoria 16, 20133 Milano, Italy \\ gioacchino.ranucci@mi.infn.it
}

G. Bellini, J. Benziger, D. Bick, G. Bonfini, D. Bravo, M. Buizza Avanzini, B. Caccianiga, L. Cadonati, F. Calaprice, P. Cavalcante, A. Chavarria, A. Chepurnov, D. D'angelo, S. Davini, A. Derbin, A. Empl, A. Etenko, K. Fomenko, D. Franco, C. Galbiati, S. Gazzana, C. Ghiano, M. Giammarchi, M. Goeger-Neff, A. Goretti, L. Grandi, C. Hagner, E. Hungenford, Al. Ianni, An. Ianni, V. Kobychev, D. Korablev, G. Korga, D. Kryn, M. Laubenstein, T. Lewke,

E. Litvinovich, B. Loer, F. Lombardi, P. Lombardi, L. Ludhova, G. Lukyanchenko, I. Machulin, S. Manecki, W. Maneschg, G. Manuzio, Q. Meindl, E. Meroni, L. Miramonti, M. Misiaszek, R. MLlenberg, P. Mosteiro, V. Muratova, L. Oberauer, M. Obolensky, F. Ortica, K. Otis,

M. Pallavicini, L. Papp, L. Perasso, S. Perasso, A. Pocar, R. S. Raghavan, G. Ranucci, A. Razeto, A. Re, A. Romani, N. Rossi, R. Saldanha, C. Salvo, S. SchÖnert, H. Simgen, M. Skorokhvatov, O. Smirnov, A. Sotnikov, S. Sukhotin, Y. Suvorov, R. Tartaglia, G. Testera, D. Vignaud, R. B. Vogelaar, F. Von Feilitzsch, J. Winter, M. Wojcik, A. Wright, M. Wurm, J. Xu, O. Zaimidoroga, S. Zavatarelli and G. Zuzel

(Borexino Collaboration)

Published 15 May 2014

\begin{abstract}
Low energy neutrino investigation has been one of the most active fields of particle physics research over the past decades, accumulating important and sometimes unexpected achievements. In this work some of the most recent impressive successes will be reviewed, as well as the future perspectives of this exciting area of study.
\end{abstract}

Keywords: Solar neutrinos; geoneutrinos; supernova neutrinos.

\section{Introduction}

Under the broad characterization of the title of this talk three very active areas of neutrino physics are considered: solar neutrinos, geo-neutrinos and supernova neutrino detection. In the following I will characterize the current status and past achievements of each field, sketching, as well, the relevant perspectives in the imminent and long term future.

This is an Open Access article published by World Scientific Publishing Company. It is distributed under the terms of the Creative Commons Attribution 3.0 (CC-BY) License. Further distribution of this work is permitted, provided the original work is properly cited. 
Solar neutrinos research is a mature field of investigation which over the past four decades has accumulated fundamental insights in the particle physics arena. Originally conceived as a powerful tool to deeply investigate the core of our star, solar neutrinos underwent a very successful detour to particle physics, heavily concurring to the successful demonstration of the neutrino oscillation phenomenon, according to the MSW mechanism. ${ }^{1,2}$ The solar experiments were also paramount to determine with high accuracy the parameters $\Delta m_{12}^{2}$ and $\sin ^{2} \theta_{12}$ of the PMNS mixing matrix governing the oscillation in the solar sector. ${ }^{3}$ Hence this extremely rich and successful chapter of particle physics produced a large part of the solid foundations upon which the next era of precision measurements of the neutrino oscillation parameters will be built. Furthermore, once completed the mission of unveiling the oscillating nature of neutrinos, solar experiments are now back to the original concept of testing the functioning mechanism of the Sun; in this context important insights are awaited from the running and future experiments, of special relevance to address the current issue regarding the surface metallic content of the Sun. ${ }^{4}$

Geoneutrino science, instead, is a much more recent experimental field, though rich of profound potential implications. The suggestion of the possible detection of antineutrino from the intrinsic radioactivity of the Earth dates back to the early times of the first neutrino detection from Cowan and Reines and is due to Gamow. Only 50 years later the solar neutrino experiment Borexino and the reactor antineutrino experiment KamLAND ${ }^{5,6}$ provided the first, and for the moment the only, indications of the observation of antineutrinos from Earth. These pioneering measurements, proving the technical feasibility of geoneutrino detection, demonstrate the practical possibility of the ambitious goal to use the measurements of the antineutrino flux to probe the characteristics of the interior of our planet.

The observation of the neutrino burst from the Supernova 1987A, performed by the Kamiokande, ${ }^{7}$ IMB $^{8}$ and Baksan ${ }^{9}$ detectors, represented in the last century a fundamental breakthrough: for the first time the last moments of a dying star were observed through the copious neutrino flux which accompanies such a cosmic thunderstorm. After that, a great deal of studies showed how the accurate determination of the supernova neutrinos can represent an extraordinarily powerful tool to investigate the evolution of the supernova mechanism; this led to the establishment of an ambitious program for the efficient capture of neutrinos from a galactic star explosion, which not only sees a growing number of experiments potentially able to perform such a task, but also their strategic alliance in a worldwide network, SNEWS, which will greatly enhance the probability of a successful detection of the eagerly awaited "galactic firework", when it will occur.

\section{Solar Neutrinos}

It is now well known, after several decades of theoretical and experimental investigations, that neutrinos are abundantly produced in the core of the Sun. They originate 
from the nuclear reactions that power our star, producing the energy required to sustain it over the billions of years of its life. Two different chain reactions occur at the temperatures characteristic of the core of the Sun, the so called $p p$ chain and CNO cycle, respectively. Actually, in the case of the Sun the vast majority of the energy $(>98 \%)$ comes from the pp chain, while the CNO contribution is estimated to less than $1.6 \%$.

The effort to produce a model able to reproduce fairly accurately the solar physical characteristics, as well as the spectra and fluxes of the several produced neutrino components, was led for more than forty years by John Bahcall; ${ }^{10}$ this effort culminated in the synthesis of the so called Standard Solar Model (SSM), which represents a true triumph of the physics of $\mathrm{XX}^{\text {th }}$ century, leading to extraordinary agreements between predictions and observables. Such a beautiful concordance, however, has been somehow recently spoiled as a consequence of the controversy arisen regarding the surface metallic content of the Sun, stemming from a more accurate 3D modeling of the Sun photosphere. Therefore, there are now two versions of the SSM, according to the adoption of the old (high) or revised (low) metallicity of the surface. ${ }^{11}$

¿From the experimental side, solar neutrino experiments also represent a successful 40 years long saga, commenced with the pioneering radiochemical experiments, i.e Homestake, Gallex/GNO and Sage, continued with the Cerenkov detectors Kamiokande/Super-Kamiokande in Japan and SNO in Canada, and with the last player which entered the scene, Borexino at the Gran Sasso Laboratory, which introduced in this field the liquid scintillation detection approach.

It is well known that for more than 30 years the persisting discrepancy between the experimental results and the theoretical predictions of the Solar Model formed the basis of the so called Solar Neutrino Problem, which in the end culminated with a crystal clear proof of the occurrence of the neutrino oscillation phenomenon, via the MSW effect. In particular, the joint analysis of the results from the solar experiments and from the KamLAND antineutrino reactor experiment pin points with high accuracy the values of the oscillations parameter within the LMA (large mixing angle) region of the MSW solution. ${ }^{3}$

So, with this spectacular conclusion in background, which surely makes the solar neutrino study one of the more productive particle physics area over the past two decades, in the following I will illustrate the peculiarity and the specific results of the two still running experiments, Borexino and Super-Kamiokande, plus those of SNO which has released until recently data from its data taking stopped in 2006, emphasizing throughout the description the perspectives of potential further achievements in the field, too.

\subsection{Borexino}

Borexino at the Gran Sasso Laboratory ${ }^{12}$ is a scintillator detector which employs as active detection medium 300 tons of pseudocumene-based scintillator. The intrinsic high luminosity of the liquid scintillation technology is the key toward the goal of 
Borexino, the real time observation of sub-MeV solar neutrinos through through elastic scattering off the electrons of the scintillator, being the ${ }^{7} \mathrm{Be}$ component the main target. However, the lack of directionality of the method makes it impossible to distinguish neutrino-scattered electrons from electrons due to natural radioactivity, thus leading to the other crucial requirement of the Borexino technology, e.g. an extremely low radioactive contamination of the detection medium, at fantastic unprecedented levels.

The active scintillating volume is observed by 2212 PMTs located on a $13.7 \mathrm{~m}$ diameter sphere and is shielded from the external radiation by more than 2500 tons of water and by 1000 tons of hydrocarbon equal to the main compound of the scintillator (pseudocumene), to ensure zero buoyancy on the Inner Vessel containing the scintillator itself. Of paramount importance for the success of the experiment are also the many purification and handling systems, which were designed and installed to ensure the proper manipulation of the fluids at the extraordinary purity level demanded by Borexino.

When data taking started in May 2007, it appeared immediately that the daunting task of the ultralow radioactivity was successfully achieved, representing per se a major technological breakthrough, opening a new era in the field of ultrapure detectors for rare events search.

The exceptional purity obtained implies that, once selected by software analysis the design fiducial volume of 100 tons and upon removal of the muon and muoninduced signals, the recorded experimental spectrum is so clean to show spectacularly the striking feature of the ${ }^{7} \mathrm{Be}$ scattering edge, i.e. the unambiguous signature of the occurrence of solar neutrino detection.

For the quantitative extraction of the ${ }^{7} \mathrm{Be}$ flux, the spectrum is fitted to a global signal-plus-background model. The latest ${ }^{7}$ Be result has been published in: ${ }^{13}$ taking into accounts the systematic errors, stemming essentially from the uncertainty in the energy scale and in the fiducial volume selection, the ${ }^{7} \mathrm{Be}$ evaluation is $46 \pm 1.5$ (stat) ${ }_{-1.6}^{+1.5}$ (sys) counts/day/100 tons, hence, summing quadratically the two errors, a remarkable $5 \%$ global precision has been achieved in this critical measurement.

By assuming the MSW-LMA solar neutrino oscillations, the Borexino result can be used to infer the ${ }^{7} \mathrm{Be}$ solar neutrino flux. Using the oscillation parameters from, ${ }^{14}$ the detected ${ }^{7} \mathrm{Be}$ count rate corresponds to a total flux of $(4.84 \pm 0.24) \cdot 10^{9} \mathrm{~cm}^{-2}$ $\mathrm{s}^{-1}$, very well in agreement with the prediction of the Standard Solar Model. ${ }^{11}$ For comparison, the measured count rate in case of absence of oscillations would have been $74 \pm 5.2$ counts/day/100tons. The resulting electrons survival probability at the ${ }^{7}$ Be energy is $P_{e e}=0.51 \pm 0.07$.

In the low energy regime the unprecedented Borexino background has allowed also the experimental study of the pep and CNO components, a possibility unanticipated during the design of the detector. By far the most important residual background for these solar neutrino fluxes is the ${ }^{11} \mathrm{C}$ decay, a radionuclide continuously produced in the scintillator by the cosmic muons surviving through the rock 
overburden and interacting in the liquid scintillator. The beta plus decay of ${ }^{11} \mathrm{C}$ originates a continuous spectrum which sits exactly in the middle of the energy region between 1 and $2 \mathrm{MeV}$, which is just the window for the pep and CNO investigation. Actually, to a less extent also the external background induced by the gammas from the photomultipliers is an obstacle, especially above $1.7 \mathrm{MeV}$.

In the reference ${ }^{15}$ a threefold coincidence strategy encompassing the parent muon, the neutron(s) emitted in the spallation of the muon on a ${ }^{12} \mathrm{C}$ nucleus, and the final ${ }^{11} \mathrm{C}$ signal has been devised and described in detail. Such a strategy applied to the Borexino data led to a pep rate of $3.13 \pm 0.23$ (stat) \pm 0.23 (sys) counts per day/100 ton, ${ }^{16}$ from which the corresponding flux can be calculated, assuming the current MSW-LMA parameters, as $\Phi($ pep $)=(1.6 \pm 0.3) \cdot 10^{8} \mathrm{~cm}^{-2} \mathrm{~s}^{-1}$, in agreement with the SSM: indeed the ratio of this result to the SSM predicted value is $f_{p e p}=1.1 \pm 0.2$. The resulting electrons survival probability at the pep energy is $P_{e e}=0.51 \pm 0.07$; it should be underlined that the significance of the pep detection is at the $97 \%$ C.L.

The same analysis, keeping the pep flux fixed at the SSM value, originates a tight upper limit on the CNO flux, i.e. $\Phi(\mathrm{CNO}) \leq 7.4 \cdot 10^{8} \mathrm{~cm}^{-2} \mathrm{~s}^{-1}$, corresponding to a ratio with the SSM prediction less than 1.4 .

The experiment has been in condition, as well, to provide important, additional insights for the higher energy ${ }^{8} \mathrm{~B}$ component. The distinctive feature of the ${ }^{8} \mathrm{~B}$ neutrino flux measurement performed by Borexino ${ }^{17}$ is the very low $3 \mathrm{MeV}$ threshold attained, decisively lower than the previous measurements from the Cerenkov experiments.

The measurement is very difficult, since the total background, both of radioactive and cosmogenic origin, in the raw data is overwhelming if compared to the expected signal. The specific background suppression strategy adopted in this case is based on two ingredients: on one hand a careful MC evaluation of the main radioactive contaminants of relevance for this measure, i.e. ${ }^{214} \mathrm{Bi}$ from Radon and the external ${ }^{208} \mathrm{Tl}$ from the nylon wall of the Inner Vessel, and on the other the "in-situ" identification and suppression of the muon and associated cosmogenic signals.

The observed ${ }^{8} \mathrm{~B}$ rate in the detector is $0.217 \pm 0.038$ (stat) \pm 0.008 (sys) cpd/100 ton, corresponding to an equivalent flux $\Phi\left({ }^{8} \mathrm{~B}\right)=(2.4 \pm 0.4 \pm 0.1) \cdot 10^{6} \mathrm{~cm}^{-2}$ $\mathrm{s}^{-1}$; if, as for the other components, we take into account the oscillation probability, then the ratio with the flux foreseen by the SSM is $0.88 \pm 0.19$.

Over the next years of running of the detector, the solar neutrino program will be completed: ${ }^{7}$ Be can be pin pointed to an accuracy of $3 \%$ (with respect to the $5 \%$ uncertainty of the measurement reported here), providing the final, high accuracy, low energy validation of the MSW-LMA solution; moreover the statistics of the ${ }^{8} \mathrm{~B}$ neutrino study will be further increased, the precision of the pep evaluation will be enhanced and an even tighter upper limit on the CNO will be placed. Furthermore, the extremely low ${ }^{14} \mathrm{C}$ level in the liquid scintillator, coupled to the good achieved energy resolution, opens also a possible exploration window between 200 
and $240 \mathrm{keV}$ in which the observation of the fundamental pp flux can be attempted: therefore Borexino holds the promise to be the first detector able to perform the complete spectroscopy of the solar neutrino flux.

\subsection{SNO (Solar Neutrino Observatory)}

While SNO has terminated its operations in 2006, the re-analysis of the recorded data has also recently produced interesting outcomes.

Succinctly, it can be reminded that this detector, located underground in Canada, in the Inco mine at Sudbury, employed heavy water to perform concurrently a neutral current all-flavor measurement, and a charged current electron-neutrino specific measurement: the comparison of the two provided the unambiguous demonstration of the occurrence of the neutrino flavour conversion process. This achievement is the collective output of the three phases in which the experiment evolved, characterized by different detection procedures of the neutrons signalling the occurrence of the neutral current reaction: a pure heavy water phase, a salt phase and the final ${ }^{3} \mathrm{He}$ counters stage.

The estimated neutral current, charged current and elastic scattering fluxes over the three phase are statistically in agreement, with the exception of the elastic scattering measure of the ${ }^{3}$ He stage, lower than the previous results, but consistent with being a downward statistical fluctuation. Also, in the latest ${ }^{3} \mathrm{He}$ measurement procedure the ratio $\mathrm{NC} / \mathrm{CC}$ resulted equal to $0.301 \pm 0.033$, slightly lower than the previous phases. While including these updated data in the global solar + KamLAND analysis, the SNO collaboration finds $\Delta m^{2}=7.46_{-0 ; 19}^{+0.20} \cdot 10^{-5} \mathrm{eV}^{2}$, and $\tan ^{2} \theta_{12}=0.427_{-0.024}^{+0.027}{ }^{3}$ It has to be noted, in particular, that the error on the $\theta_{12}$ angle has been reduced of almost a factor two by the ${ }^{3} \mathrm{He}$ measurement, as effect of the different systematic and minimal correlation of the $\mathrm{NC}$ and $\mathrm{CC}$ measures in this phase.

The recent LETA (Low Energy Threshold Analysis) re-processing of the data has further pushed down the analysis threshold, pursuing (similarly to SuperKamiokande, see next sub-paragraph) the investigation of the survival probability in the $4-5 \mathrm{MeV}$ region, with the aim to unravel the up-turn, if any, of the ${ }^{8} \mathrm{~B}$ spectrum. Such an up-turn is expected as the imprint of the MSW effect in that specific energy region. The result stemming from this analysis is definitively puzzling, since it incredibly seems to point event to a down-turn of the spectrum.

This outcome, coupled to the absence of the up-turn in the Super-Kamiokande results, points to something new lurking in that energy region. Additional high statistic data, however, will be needed to understand what is happening there. In this respect, also the more precise evaluation of the pep rate that Borexino plans to perform within a couple of years will be of fundamental importance.

\subsection{Super-Kamiokande}

The Cerenkov experiment Super-Kamiokande, still taking data, has recently released new results about the ${ }^{8} \mathrm{~B}$ flux obtained with threshold lowered to $3.5 \mathrm{MeV}$. 
The long history of this detector started in 1996 and evolved through four phases: the first phase lasted until a major PMT incident in November 2001 and produced the first accurate measurement of the ${ }^{8} \mathrm{~B}$ flux via the $\mathrm{ES}$ detection reaction. The phase II with reduced number of PMTs, from the end of 2002 to the end 2005, confirmed with larger error the phase I measurement. After the refurbishment of the detector back to the original number of PMTs, the third phase lasted from the middle of 2006 up to the middle of 2008. After that, an upgrade of the electronics brought the detector into its fourth phase, which contemplates also the accelerator neutrino beam experiment $\mathrm{T} 2 \mathrm{~K}$. It is important to highlight the evolution of the energy threshold (total electron energy) in all the phases: $5 \mathrm{MeV}$ in phase I, $7 \mathrm{MeV}$ in phase II, $4.5 \mathrm{MeV}$ in phase III and $3.5 \mathrm{MeV}$ in phase IV, thanks to the continuously on-going effort to reduce the radon content in water.

The important result provided by Super-Kamiokande is the value of the equivalent ${ }^{8} \mathrm{~B}$ flux, obtained converting the all flavor ES measure into an effective neutrino flux without correcting for the oscillation probability; the Super-Kamiokande IV precise measurement amounts to $2.32 \pm 0.02$ (stat) \pm 0.04 (sys) $\cdot 10^{6} \mathrm{~cm}^{2} \mathrm{~s}^{118}$ representing a high accuracy confirmation that the electron neutrino flux, which contributes mostly to the result of the experiment, is drastically reduced (about 60\%) with respect to the SSM prediction.

The recent release of low threshold data had also the goal to unravel the existence of the low energy, eagerly sought, up-turn in the ${ }^{8} \mathrm{~B}$ spectrum. However, the upturn did show up only mildly, the experimental spectrum above $3.5 \mathrm{MeV}$ showing only a $1 \sigma$ preference for the distortion predicted by standard neutrino flavour oscillation parameters over a flat suppression. In this respect, therefore, the poor definition of the data around $3.5-4 \mathrm{MeV}$ and the contradictory output of SNO call for additional evaluations. More data, hence, are needed to further shed light about this point.

\subsection{Physics implications}

In Fig. 1 from Ref. 19 the MSW predicted $P_{e e}$ (electron neutrino survival probability, grey band) is shown, together with several experimental points, i.e. black the ${ }^{8} \mathrm{~B}$ from SNO and Super-Kamiokande, green ${ }^{8} \mathrm{~B}$ from Borexino and SNO Low Threshold Analysis, blue the ${ }^{7} \mathrm{Be}$ Borexino point, magenta the $p p$ datum as drawn by the comparison of Borexino with the Gallium experiments, and red the pep Borexino point: altogether from this figure we can conclude that the solar data collectively accumulated so far spectacularly confirm over the entire energy range of interest the MSW-LMA solar neutrino oscillation scenario, providing the clear evidence of the transition from the high energy, more suppressed "matter" regime, to the low energy, less suppressed "vacuum" regime.

Furthermore, all the solar and KamLAND data taken together constrain rather precisely the oscillation parameters. Fig. 2, from Ref. 3, shows the allowed region in the parameter space stemming from a two flavor oscillation analysis. In a first 


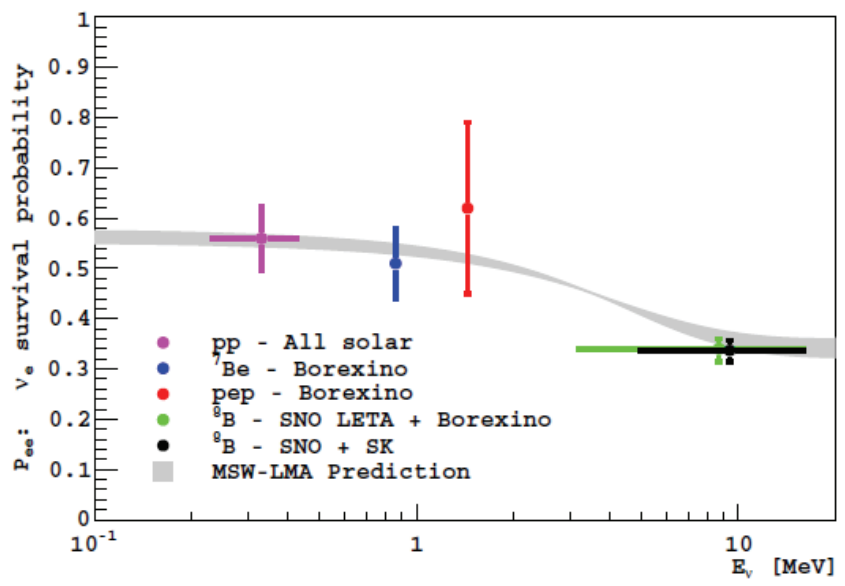

Fig. 1. Low energy validation of the MSW-LMA solution provided by all solar data.

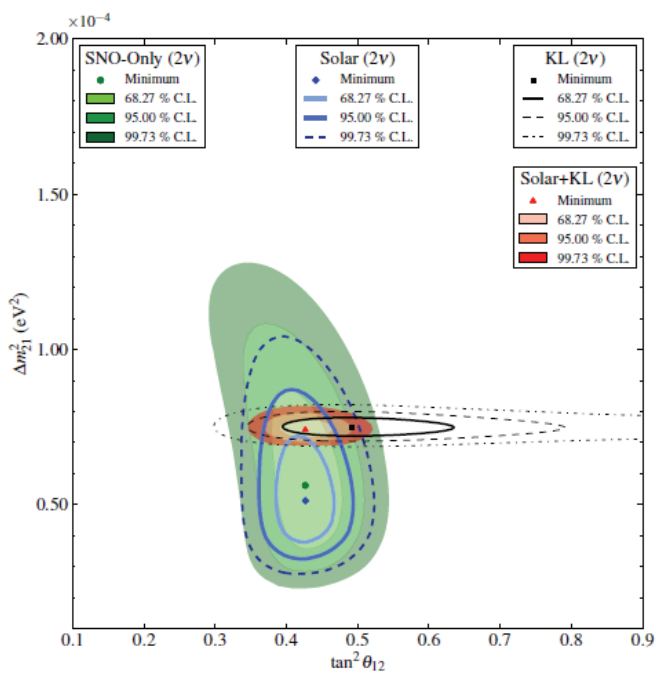

Fig. 2. Allowed region of the oscillation parameter plane.

approximation, the strongest constraint on the $\Delta m^{2}$ parameter comes from KamLAND, while the limit on the mixing angle derives from the solar data.

\subsection{Outlooks}

Despite the high degree of maturity reached by the field, solar neutrino investigation still has interesting future perspectives. The two current generation experiments continuing with data taking, Borexino and Super-Kamiokande, will go ahead 
along the lines outlined above: Borexino will attempt the high precision, full solar spectroscopy while Super-Kamiokande will accumulate more solar data at lower threshold to try to shed light on the "mystery" of the missing up-turn.

Looking ahead to the near future, $\mathrm{SNO}+{ }^{20}$ is the new experiment that will replace SNO. It is a liquid scintillator detector, like Borexino, that can go on line soon thanks to the massive re-use of the SNO hardware. In particular the adoption of a new liquid scintillator, linear alkyl benzene, featuring the great advantage of being acrylic-compatible, will allow the re-use of the SNO acrylic vessel. Only the support system had to be built from scratch in order to cope with the different buoyancy condition with respect to the heavy water situation. The other new major piece of equipment that has been realized is the scintillator purification system. The project has been funded for these major items on June 2009; all the planned refurbishments have been completed, and a preliminary operation of water fill is now in progress. The solar program of the experiment will be targeted mainly to pep and CNO detection, profiting of the depth of SNOLAB, which suppress enormously the cosmogenic ${ }^{11} \mathrm{C}$ background. It should be, however, highlighted that the main goal of SNO+ will be the double beta decay with Tellurium dissolved in the scintillator, and currently it is not yet decided whether the solar phase will come before or after the Tellurium phase. The data taking with scintillator will start within 2014.

Far in the future there may be experiments based on completely new techniques like for example LENS, ${ }^{21}$ which is the modern version of the old brilliant idea for an Indium experiment. The experimental tool used for the detection of solar neutrinos is the tagged capture of electron-flavor neutrinos on ${ }^{115} \mathrm{In}$ via charged current: $\nu_{e}+{ }^{115} \mathrm{In} \rightarrow{ }^{115} \mathrm{Sn}^{*}+\mathrm{e}^{-} \rightarrow{ }^{115} \mathrm{Sn}+2 \gamma$. The low $Q$ of the reaction $(114 \mathrm{keV})$ allows in principle the full solar neutrino spectroscopy, including the $p p$ neutrinos. Time and space coincidence, the former intrinsic to the detection reaction and the latter realized via a granular detector design, will be the key to suppress the radioactive backgrounds as well as the inherent background from the beta decay of ${ }^{115} \mathrm{In}$.

Finally, it should be mentioned that massive cryogenic detectors for dark matter search based on liquefied noble gases, if realized with the mass of several tens of tons of material, can be perfectly suited for the precise pp spectroscopy. This is true in particular for detector based on Neon and Xenon, which are exempted from low energy radioactive isotopes; this is not the case of Argon due to the presence of the radioactive ${ }^{39} \mathrm{Ar}$.

\section{Geoneutrinos}

Geoneutrinos, the antineutrinos from the progenies of $\mathrm{U}$, Th and ${ }^{40} \mathrm{~K}$ decays in the Earth, bring to the surface information from the whole planet, concerning its content of radioactive elements. Their detection can shed light on the sources of the terrestrial heat flow, on the present composition and on the origins of the Earth. 
Although geo-neutrino detection was conceived very long ago, only recently they have been considered seriously as a new probe of our planet, as a consequence of two fundamental advances that occurred in the last few years: the development of extremely low background neutrino detectors and the progress on understanding neutrino propagation.

Geoneutrinos potentially can unlock many interesting unanswered questions regarding our planet: what is the radiogenic contribution to terrestrial heat production? How much is the content of $\mathrm{U}$ and $\mathrm{Th}$ in the crust and in the mantle, respectively? What is hidden in the Earth's core, e.g a geo-reactor or ${ }^{40} \mathrm{~K}$, etc.? And finally, is the standard geochemical model (denoted as BSE) consistent with geo-neutrino data?

In particular this last question would ideally require multiple measurements carried out in several locations of the Earth's surface, to be compared with the theoretical predictions of geoneutrino flux (different groups performed such calculations, which are each other in agreement at the $10 \%$ level $^{22-24}$ ).

The detection occurs via the classical inverse beta reaction $\bar{\nu}_{e}+p \rightarrow e^{+}+n$, signaled by the delayed $2.2 \mathrm{MeV}$ gamma stemming from the subsequent neutron capture on protons, for which the most important background is represented by anti-neutrinos from reactors. Exploiting this approach, Borexino and KamLAND ${ }^{5,6}$ are the experiments that so far provided the first geo-neutrino measurements.

In particular the latest geoneutrino data from Borexino has been reported in Ref. 5, for a data taking period of 1353 days. With a fiducial exposure of (3.69 \pm 0.16$)$. $10^{31}$ proton $\times$ year, after all selection cuts and background subtraction, $(14.3 \pm 4.4)$ geo-neutrino events were detected, assuming a fixed chondritic Th/U ratio of 3.9. This result corresponds to a geoneutrino signal of $(38.8 \pm 12.0)$ TNU (Terrestrial Neutrino Unit: $=1$ event $/$ year $/ 10^{32}$ protons), disfavouring also the no-geoneutrino hypothesis with a $p$-value of $6 \times 10^{-6}$.

The reactor neutrino background was evaluated equal to $31.2_{-6.1}^{+7}$, well in agreement with the expectation $(33.3 \pm 2.4)$, thus confirming the validity of the entire measurement procedure.

The updated KamLAND results in Ref. 6 are in line with the Borexino output, amounting to $(31.1 \pm 7.3)$ TNU.

The main virtue of the pioneering results of Borexino and KamLAND is on one hand the demonstration that geoneutrinos can be detected, paving the way to a completely new branch of geosciences, and on the other that there is a broad agreement with the predictions of the standard BSE model of the composition of the Earth. Obviously, the low statistics of both measures hampers the possibility to distinguish among variants of this general model. This is left for future massive detectors: in particular after the imminent $\mathrm{SNO}+$, which will complement the Borexino and KamLAND data with measurements of similar statistical size, the two planned gigantic detectors JUNO and LENA ${ }^{25,26}$ will have the capability of several hundred events per year. 


\section{Supernova Neutrinos}

On February 23, 1987 the Kamiokande, ${ }^{7} \mathrm{IMB}^{8}$ and Baksan ${ }^{9}$ detectors recorded for the first (and up to now the only) time a burst of neutrinos from a supernova, the famous SN1987A. Despite the small number of recorded signals, 11, 8 and 5 respectively, plenty of information was squeezed from them, providing substantial confirmations and new insights on the supernova mechanism. On the basis of this success, it can be anticipated that further deep insights and details of the supernova origin and evolution will be gained when the next burst will be observed, especially if the supernova will occur at the typical galactic distance from the Earth of $10 \mathrm{kpc}$, compared to the $50 \mathrm{kpc}$ distance of the SN1987A, which indeed was located in the Large Magellanic Cloud.

A number of multipurpose detectors, some already running and some still in the construction phase, will detect the next supernova neutrinos. Among the current detectors, Super-Kamiokande is surely the largest with its 32000 tons of water. For a $10 \mathrm{kpc}$ distant supernova it will observe about $7300 \bar{\nu}_{e}+p \rightarrow e^{+}+n$ events, $\sim 300 \nu+e \rightarrow \nu+e$ scattering events, $\sim 360{ }^{16} \mathrm{O}$ neutral current gamma events and $\sim 100{ }^{16} \mathrm{O}$ charged current events. The scattering events will identify the supernova direction with an accuracy of about $5^{\circ}$.

The large statistics of inverse beta events, instead, will allow the precise energy spectrum measurement, enabling the possibility to discriminate among various proposed models. Besides being the major detection channel of the liquid scintillation detectors, it may take place also in Super-Kamikande, if the current effort to dissolve gadolinium in water will be successful.

At Gran Sasso since 1992 the LVD $^{27}$ detector is in operation: it is a segmented liquid scintillator type apparatus with 840 counters and a total target mass of 1000 tons. It can detect about $300 \bar{\nu}_{e}+p \rightarrow e^{+}+n$ events for the reference $10 \mathrm{kpc}$ supernova. The energy threshold of the counter is $4 \mathrm{MeV}$ and it can tag neutrons with an efficiency of $50 \pm 10 \%$. The online trigger efficiency is more than $90 \%$ for a distance less than $40 \mathrm{kpc}$.

KamLAND, Borexino and the future SNO+ with their 1000, 300 and 1000 tons of detection medium, respectively, are perfect supernova observatories. They feature several interactions channels: by normalizing the expected number of events for a 10 kpc supernova to a target mass of 1000 tons, the predictions are $\sim 300 \bar{\nu}_{e}+p \rightarrow e^{+}+n$ events, several tens of $\mathrm{CC}$ and $\mathrm{NC}$ events on ${ }^{12} \mathrm{C}$ and about $300 \nu+p \rightarrow \nu+p$ NC events. This last interaction, being not affected by neutrino oscillations, has the very interesting capability to measure the initial spectrum originated by the supernova explosion. Clearly these numbers will increase of two order of magnitudes in the next generation, huge scintillation detectors JUNO and LENA.

The South Pole IceCube detector has a giga-ton target volume, with the photosensors arranged in a three dimensional structure. The entire inventory of strings (86) has now been deployed. In this detector the observation of a supernova burst will not occur via individual neutrino observation, but through the coherent increase 
of the PMT dark noise. As a consequence, the detector can measure the time variation of the energy release fairly accurately. ${ }^{28}$

Finally, it has to be highlighted that Super-Kamiokande, LVD, IceCube and Borexino are part of the SNEWS consortium (Supernova Early Warning System), which, upon detection of a neutrino burst, sends prompt alerts to astronomers to trigger the optical observation of the potential identified source. More detailed information on this topic can be found in Ref. 29.

\section{Conclusions}

Low energy neutrino physics and astrophysics is a field that, despite the many successes already accumulated, is promising for the future many more exciting results.

Solar neutrino investigation is reaching the stage in which the precise spectroscopy of the whole neutrino energy spectrum is a concrete case, up to a level that makes it feasible the check of the SSM, hence paving the path for possibly resolving the discrepancy between high $Z$ and low $Z$ models and understanding the mystery of the up-turn in the ${ }^{8} \mathrm{~B}$ spectrum.

An entire new area of investigation is represented by the geoneutrinos study, which exploits the more powerful detectors aimed at solar and reactor neutrino detection to open a completely new window on the characteristics of the interior of our planet.

Supernova neutrino experiments are already a powerful and large family, with new members expected to join in the near future, and hence if Nature will arrange for us a "galactic firework", surely we will not miss the fun!

\section{Acknowledgments}

The author wihes to thank the organizers for the invitation to contribute to such an interesting and enlightening conference.

\section{References}

1. L. Wolfenstein, Phys. Rev. D 17, 2369 (1978).

2. S.P. Mykheyev and A.Yu. Smirnov, Sov. J. Nucl. Phys. 42, 913 (1985).

3. B. Aharmim et al., arXiv:1109.0763 [nucl-ex] (2008).

4. M. Asplund et al., Annu. Rev. Astron. Astrophys. 47, 481 (2009).

5. G. Bellini et al. (Borexino Collaboration), Phys. Lett. B 722, 295 (2013).

6. A. Gando et al., Phys. Rev. D 88, id. 033001 (2013).

7. K. Hirata et al., Phys. Rev. Lett 58, 1490 (1987).

8. R. M. Bionta et al., Phys Rev. Lett. 58, 1494 (1987).

9. E. N. Alexeyev et al., Phys. Lett. B 205, 209 (1988).

10. http://www.sns.ias.edu/ jnb/ John Bahcall's home page.

11. A. M. Serenelli, W. C. Haxton, and C. Pena-Garay, Astrophys. J. 743, 24 (2011).

12. G. Alimonti et al., Nucl. Instr. and Meth. A 600, 568 (2009).

13. G. Bellini et al. (Borexino Collaboration), Phys. Rev. Lett. 107, 141302 (2011).

14. Review of Particle Physics, K. Nakamura et al. (Particle Data Group), J. Phys. G 37, 075021 (2010). 
15. H. Back et al. (Borexino Collaboration), Phys. Rev. C 74, 045805 (2006).

16. G. Bellini et al. (Borexino Collaboration), Phys. Rev. Lett. 108, 051302 (2012).

17. G. Bellini et al. (Borexino Collaboration), Phys. Rev. D 82, 033006 (2010).

18. H. Sekiya, for the Super-Kamiokande Collaboration, proceedings of the $33^{\text {rd }}$ International Cosmic Ray Conference, Rio De Janeiro 2013 (ICRC2013) arXiv:1307.3686 [hep-ex].

19. G. Bellini et al. (Borexino Collaboration), arXiv:1308.0443 [hep-ex] (2013).

20. M. Chen, Nucl. Phys. B (Proc. Suppl.) 154, 65 (2005).

21. R. S. Raghavan, Journal of Physics: Conference Series 120, 052014 (2008).

22. S. Enomoto, Ph.D. thesis, Tohoku University (2005).

23. G. Fogli et al., Phys. Lett. B 623, 80 (2005).

24. F. Mantovani et al., Phys. Rev. D 69, 013001 (2004).

25. Y. Li et al., Phys. Rev. D 88, 013008 (2013).

26. M. Wurm et al., Astropart. Phys. 35, 685-732 (2012).

27. N. Yu. Agafonova et al., Astropart. Phys. 28, 516 (2008).

28. R. Abbasi et al., arXiv:1108.0171 [astro-ph.HE] (2011).

29. K. Schoelberg, arXiv:1205.6003 [astro-ph.IM] (2011). 